\title{
Perception Aptitude Improvement of an Odor Sensor: Model for a Biologically Inspired Nose
}

\author{
B. Lorena Villarreal ${ }^{1}$ and J.L. Gordillo ${ }^{2}$ \\ Center for Robotics and Intelligent Systems, Tecnológico de Monterrey \\ Monterrey, Nuevo León, México \\ lvillarreal.bg@gmail.com, jlgordillo@itesm.mx
}

\begin{abstract}
Biologically inspired systems are a common tendency in robotics. Nowadays the common robots use human-like behaving senses as capabilities as soon as they can see, hear and touch, but the senses of smell and taste, are starting to emerge. There are three main problems to solve when including a smell sensor into a robot: the environmental model or the way the odor molecules behave either in outdoors or indoors, the sensor model, and the algorithmic or process model. One of the difficulties of developing chemical sensors versus another sensor is that chemical reactions tend to change the sensor composition often in a way that is nonreversible. Also, the odor exposure quickly saturates the sensor which needs a lot of time to be ready for the next measure. This is why; the smell system design must be biologically inspired. In this paper we present the results of the sensor model including the biological inspired process of aspiration and the design of a smell system device.
\end{abstract}

Keywords: smell, bio-inspired nose model, nose system, nostrils, smell sense, chemical sensor model.

\section{Introduction}

Biologically inspired systems are a common tendency in robotics. Nature is a wide and wise teacher of how to reach specific goals. This is why; robotics and intelligent systems are trying to emulate behaviors and physical mechanisms out of it. For example there are artificial systems using algorithms based on animal behaviors to reach cooperation and coordination [1], odor path followers [2, 3], while there are also mechanical systems learning how to walk exactly as a human would do [4].

Nowadays there are a huge variety of robots emulating human senses as capabilities to reach its goals, as soon as they commonly can see, hear and touch, but the senses of smell and taste, are not commonly implemented. Although olfaction seems not to be essential for our survival, in fact, it is very important because if we lost this sense our lives becomes a little more dangerous, for example, we would not be alerted to spoiled foods, leaking gas or smoke from a fire [5].

In animal kingdom it is used for inspection, recognition, mating, hunting, and others. Due to this ability, dogs smell capability can be trained to search for drugs, explosives, some chemicals or hazardous substances or even lost people and are commonly 
used in search and rescue operations at disaster areas, at airports or border controls [6]. Smell sensors and robotics are starting to emerge as a unified area using emulations of the fast response of hound dogs in search and rescue operations [7-9]. At this task, the principal problem is the odor source localization. Nevertheless, to obtain the best algorithmic results, the perception of the environment is crucial.

There are three main problems to solve when including a smell sensor into a robot: the environmental model or the way the odor molecules behave either in outdoors or indoors, the sensor model, and the algorithmic or process model.

The main characteristics of commonly used smell sensors are the discrimination between odors and the sensitivity [10]. One of the difficulties of developing chemical sensors versus another sensor is that chemical reactions tend to change the sensor composition often in a way that is nonreversible. Also, the odor exposure quickly saturates the sensor which needs a lot of time to be restarted. There are some researchers trying to solve the saturation problem by other solutions [11].

Knowing that odor can be propagated without air flows present by diffusion, and by advection depending on the laminar air velocity, in a non-reversible process that increases the entropy, as the chemical reactions take place [12]; in this paper we are focusing on the perception problem, showing the results of the mathematical model for the sensor that includes as improvement the addition of the aspiration process biologically inspired, and the qualitative model for the sensorial system design.

In the following section we present the related work about smell and robotics applications, then on the third section the analysis of the biological nose, followed by the analysis of the bio-inspired sensorial model and the mathematical sensor model in section 4. In section 5 are presented the results. Finally on section 6 we present the conclusions and future work.

\section{Related Background}

\subsection{Chemical Sensors Applications}

Pashami et. al. in [13] propose a change point detection algorithm to find chances in intensity, compound, or mixture ratio of an odor. In this approach they evaluate it on individual gas sensors in an experimental setup where a gas source changes these variables.

But besides the discrimination of an odor, chemical sensors can be useful in a lot of more applications, for example: Using the concentration measurement at continuous time, the increment or decrement of certain odor could alert an oil plant about some leak of toxic or explosive gas and the corresponding time averaged behavior of it. This cannot be achieved if the sensor is saturated with a peak of that certain odor. This could lead to a false alarm and probably to a loss of time or money. In the other hand, the constant minimum increment that chemical sensors produce innately could lead to a constant replacement. In these applications it is important that odor sensors had the capability of desaturation.

The saturation of the sensor is a common problem in chemical sensors. Some research is focused to solve it [11]. They are using a multi-chamber system to decrease 
the sampling time between sensors, but instead of it, we are trying to simplify the design by using only one chamber.

\subsection{Smell and Robotics Applications}

The principal task of a sniffing robot is the odor source localization. The algorithms used to solve this problem are commonly categorized by the terms of chemotaxis and anemotaxis. The first one refers to the orientation and movement of the agent in relation to the chemical gradient. In the other hand, anemotaxis, instead of following the gradient, considers the direction or current of a fluid [14].

Ishida and Miyatani in [15] are using pumps pulling air into a chamber with chemical sensors and creating an air curtain between two of them to produce two samples at different positions at the same time.

In [16] an odor grip map was presented, where the readings of the robot are convolved as soon as it moves using the radial symmetric two dimensional Gaussian function to create the next portion of the grid map. They used three different robot movement techniques: spiral, sweeping and instant gradient.

From this research, a hound robot was presented by Loutfi et. al. in [9]. This robot discriminates odors and creates grid maps of these using the sweeping movement technique. A major drawback of this approach is that the volatility of the odor particles causes them to be quickly distributed in the room so, by the time the robot has finished its sweeping routine, the distribution of the odor could be different.

Lochmatter and Martinoli [3, 17] developed three bio-inspired odor source localization algorithms, using a chemical sensor. The algorithms implemented in this research have the tendency of using the wind measurements to decide the best movement of the robot. They are also working on a simulation platform for the experimentation process [2].

At this point, the analysis of the sensor model and its efficient functionality is crucial before implement the odor source localization algorithms. This is why; this research is focused in the analysis, design and implementation of a biologically inspired sensor that generates a better measurement. The next section explains the human nose process to allow a complete understanding of the proposed design.

\section{Biological Nose Functionality}

According to [18], knowledge of the detailed air flow patterns in the human nasal cavity and the subsequent quantity of odorant transport to the olfactory receptor cites appear essential to a complete understanding of human olfaction. This is why the design of a biologically inspired smell sensor must considerate the air flow patterns to simulate complete olfaction.

The nose, in both, animals and humans, has two principal functions: breathing and odor perception [19]. Each nostril or nasal cavity is divided in three zones which are the nasal airway with bones into the lateral walls of the nasal cavity, forming a series of folds called turbinates; the epithelium, surrounded by an aqueous mucus layer; and 
then the olfactory bulb is supported and protected by the cribriform plate (cartilage) which in mammals, separates it from the olfactory epithelium [20].

Odor perception starts with the transport of volatile chemical molecules (odor molecules) by air flow to the olfactory receptors inside the nose, specifically on the ciliated olfactory epithelium [21]. Then it is electrically transmitted to the olfactory bulb and ultimately to the brain [22].

The mucus helps to trap bacteria, particulate matter, and toxins. Conduction, filtration, heating and humidification are aided by mucosal and the turbinates [20].

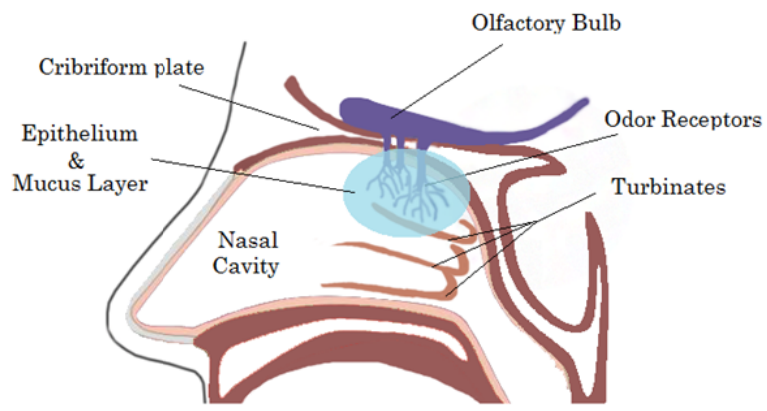

Fig. 1. Olfactory biological system of a human

There are three turbinates on each side of the nasal cavity and greatly increase the surface area of the nasal cavity. During inhalation air is directed over and under the turbinates. They are unconsciously controlled so that they swell on one side at a time and shrink on the other side. In humans, the turbinates divide the nasal airway and are responsible for forcing inhaled air to flow in a steady, regular pattern around the largest possible surface of cilia and climate-controlling tissue [20].

Upon entering the nasal cavity, inspiratory airflow is well mixed by turbulence, ensuring delivery of a representative odor sample to ephitelium [19]. It has been estimated that only $10 \%$ of inhaled air actually reaches the olfactory region during a normal resting breath [23].

This homogenized sample that reaches the odor sensors, send the electrical signal to the olfactory bulb, where data is pre-processed by glomeruli synapses [24] and the odors are discriminated. Finally the electrical information is send to the neocortex [22], where further processing takes place.

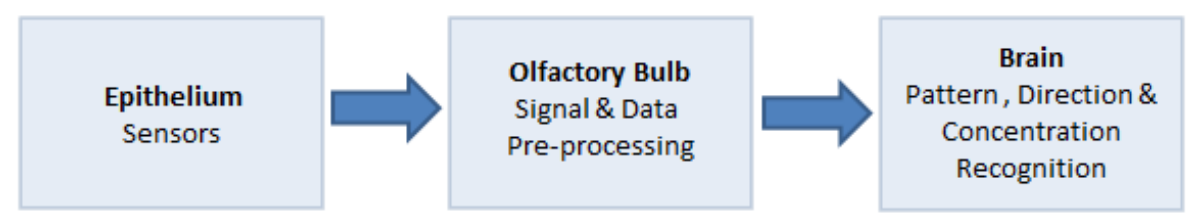

Fig. 2. Olfactory processes taking place in the biological nose 


\section{$4 \quad$ Perception System}

As said before, the intention of this paper is to prove that the biological nose system can be emulated and implemented, improving the perception aptitude of chemical sensors thanks to the aspiration process generating a desaturation capability. At this point it is important to understand the implementation and the sensor behavior. This section describes the design and sensor model with and without the aspiration process.

\subsection{Bio-inspired Design}

Knowing that the smell sense in nature is divided in stages, we implemented a biologically inspired design of a nostril $[25,26]$ :

1. Aspiration process. At this stage the odor is carried by an air flow being absorbed by a nostril. In this process there must be a physical filter to protect the sensors from dust and other particles. The humidification of the sensor is also necessary to amplify the odor.

2. Conduction. At this stage the turbinates homogenize the air and mix the odor in such a way that the mix is prepared to be sensed. Then a good sample of the odor is conducted to the sensory array. The other part of the air is used in respiration.

3. Sensing. In this stage each array consists of three sensors separated by $2.5 \mathrm{~cm}$ from center to center in a linear position.

4. Processing. This is the algorithmic stage of the smell sense. It means that the data collected is sent to the brain which analyzes the information.

5. Transforming. The air is transformed to clean the sensory system so the saturation is not occurring.

6. Exhalation. The last stage is the expulsion of the air through the nose and the ventilation of all the system.

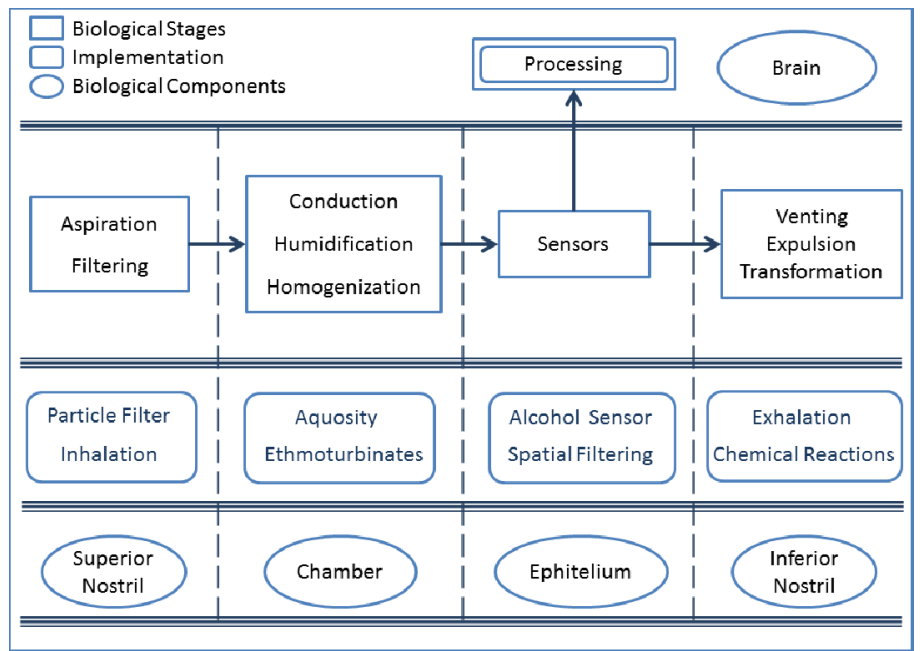

Fig. 3. Comparison between olfaction stages and processes, biological and implemented 
All these stages are included in the smell system developed in this research to provide an efficient and optimized measurement. The complete system has the ability to inhale and exhale, using two ventilators in opposite direction, one for pull the air into the chamber, and one to send it out. After the measurement, the chamber is cleaned. It has different turbinates, that transport the odors at the same time that is being mixed, and then a sample of this mix is oriented to the sensory system. This system provides the homogenization and sampling of environment besides the results in section 5 .

\subsection{Sensor Model}

The aim of a model is to represent the predicted behavior of a system in a logical, simplified and objective way depending on an input. In this research we are using an odor exposure as the input, and improving the model described in [27] by the inclusion of the aspiration process. A model is also useful to understand the behavior of the system by the analysis of the results obtained with the modification of common independent variables.

The gas sensor selected discriminates alcohol from any other odor. The chemical reaction changes the resistance between two terminals and because the supply voltage is maintained the current over the load changes and generates a different voltage on it. Then the results of the three sensors in the array for each nostril, are averaged and this average $V_{\text {in }}$ [28] is the one that would be measured and analyzed. The concentration change (\% C) was calculated as in [2]:

$$
\% C=(\Delta R) /\left(V_{\max }-R\right) 100
$$

Where $V_{\max }$ is the maximum voltage ever measured, $\mathrm{R}$ or Reference is the lower voltage ever measured in absence of alcohol, and $\Delta R$ or differential range is the difference between averaged $V_{i n}$ and $\mathrm{R}$.

$$
\begin{aligned}
& r(t)=\left\{\begin{array}{rr}
r_{1}(t) & \text { if } t<t_{s} \\
r_{2}(t) & \text { if } t_{s}<t<t_{s}+\Delta t_{r} \\
r_{3}(t) & \text { if } t_{s}+\Delta t_{r}<t<t_{s}+\Delta t_{a}+\Delta t_{r} \\
r_{4}(t) & \text { if } t_{s}+\Delta t_{r}+\Delta t_{a}<t
\end{array}\right. \\
& r_{1}(t)=\mathrm{R}_{0} \\
& r_{2}(t)=R_{0}+\left(R_{\max }-R_{0}\right)\left(1-\exp \left(\frac{-\left(t-t_{s}\right)}{\tau_{r}}\right)\right) \\
& r_{3}(t)=R_{\text {max }}^{*}+\tau_{a}\left(t-t_{s}-\Delta t_{r}\right)\left(1-\frac{\left(t-t_{s}-\Delta t_{r}\right)}{2}\right) \\
& r_{4}(t)=R^{\prime}{ }_{0}+\left(R^{*}{ }_{a}-R^{\prime}{ }_{0}\right)\left(\exp \left(\frac{-\left(t-t_{s}-\Delta t_{r}-\Delta t_{a}\right)}{\tau_{d}}\right)\right) \\
& R_{\text {max }}^{*}=R_{0}+\left(R_{\text {max }}-R_{0}\right)\left(1-\exp \left(-\Delta t_{r} / \tau_{r}\right)\right) \\
& R^{*}{ }_{a}=R_{\text {max }}^{*}+\tau_{a}\left(t_{s}+\Delta t_{r}+\Delta t_{a}\right)\left(1-\frac{\left(t_{s}+\Delta t_{r}+\Delta t_{a}\right)}{2}\right)
\end{aligned}
$$


In the complete model we have different adjustable variables: the response level before $\left(R_{0}\right)$ and after $\left(R_{0}^{\prime}\right)$ the stimulus, the saturation level $\left(R_{\max }\right)$, the maximum value reached $\left(R_{\text {max }}^{*}\right)$, the value before exhalation $\left(R^{*}\right)$, the time constants of rise $\left(\tau_{r}\right)$, sampling the air $\left(\tau_{\mathrm{a}}\right)$, and decay $\left(\tau_{\mathrm{d}}\right)$, the time before the sensor started to respond $\left(\mathrm{t}_{\mathrm{s}}\right)$, the duration of the rising period $\left(\Delta t_{r}\right)$ and the duration of the sampling period $\left(\Delta t_{a}\right)$.

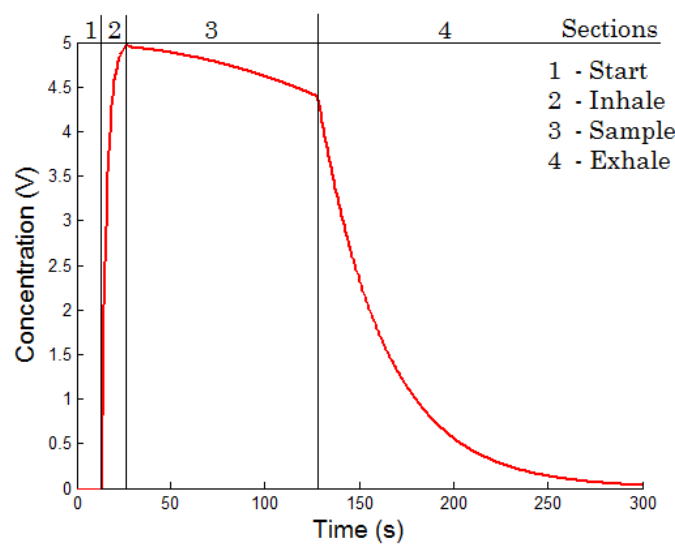

Fig. 4. Sensor model including inhalation, sampling and exhalation processes

Figure 4, displays the behavior of the sensor when an odor is exposed as a pulse, meaning that alcohol is exposed just an instant near the sensor. It is assumed an ideal first-order sensor and thus models the dynamic response to a pulse or step stimulus as an exponential rise and decay.
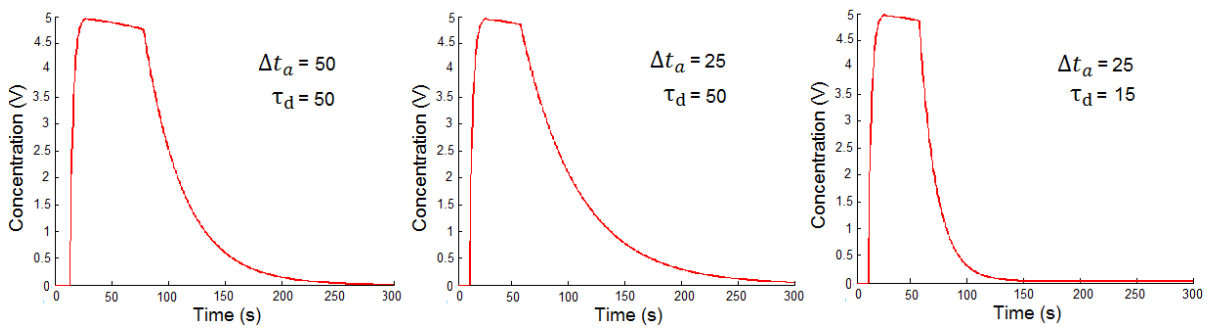

Fig. 5. Results with different sampling periods and decay time constants

It can be seen in Figure 5 that the decay time constant $\left(\tau_{d}\right)$ is not affected by decreasing the sampling period, instead, the system can sample in a short period, desaturating the sensor faster. To achieve this faster recovery of the sensors $\tau_{d}$ should be a lower value at exhalation. 


\section{$5 \quad$ Results}

The experiments were realized in a robotics lab of $5 \times 5 \mathrm{~m} 2$. The room was ambient temperature and flows of air were present in the room. The only restriction was to avoid transversal near floor currents through the path between the odor source and the sensors.
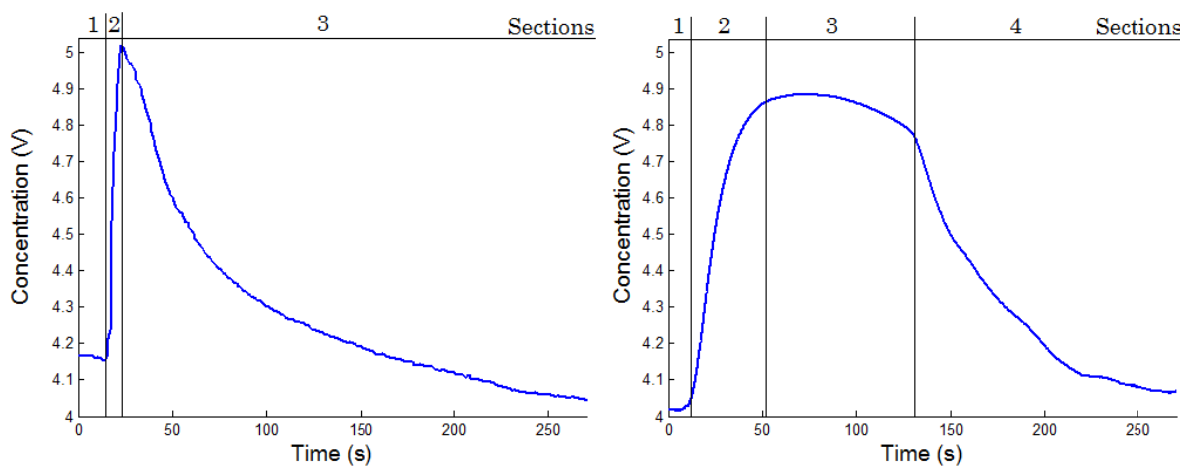

Fig. 6. Left: Sensor behavior without aspiration. Right: Sensor behavior with biologically inspired aspiration.

Three different processes were analyzed for aspiration including: inhalation, exhalation and the response when both ventilators are turned off.

The first set of experiments consisted in the comparison between the sensor with and without aspiration and smell system. Figure 6 shows the same pattern obtained by the mathematical model. It can also be noticed that desaturation process occurs faster with the aspiration model. It was obtained with the experimental data and the mathematical model a $\tau_{d}=60.23$ when no aspiration is included and $\tau_{d}=18.41$ otherwise, meaning that desaturation occurred around $70 \%$ faster with the biologically inspired nose system.

Table 1. Results of the rate of change of nostril measurements at different conditions

\begin{tabular}{cccc}
\hline $\begin{array}{c}\text { Aspiration } \\
\text { Process }\end{array}$ & Environment & Rate & Behavior \\
\hline Off & Odor Source & $3 \mathrm{mV} / \mathrm{s}$ & Slow Rise \\
Exhale & Unsaturated & $-15 \mathrm{mV} / \mathrm{s}$ & Fast Decay \\
Inhale & Odor Source & $33 \mathrm{mV} / \mathrm{s}$ & Fast Rise \\
Off & Unsaturated & $-0.3 \mathrm{mV} / \mathrm{s}$ & Static \\
Exhale & Odor Source & $-5 \mathrm{mV} / \mathrm{s}$ & Slow Decay \\
Inhale & Unsaturated & $1 \mathrm{mV} / \mathrm{s}$ & Very Slow Rise \\
\hline
\end{tabular}


Table 1 shows the comparative results of the rate of change in $\mathrm{mV} / \mathrm{s}$ of the nostril measurements when an odor source is present and the ventilation system is off, inhaling or exhaling. And the same analysis when the odor source was turned off or closed.

These results show that aspiration is crucial when a sample of the environment is analyzed into the chamber; otherwise the sensors are saturated easily. Inhalation pull air into the nostril and the measure is reached faster, and exhalation desaturates the sensor even when an odor source is saturating the environment as a normal leak of gas.

\section{Conclusions and Future Work}

Thanks to the nostril design, we were able to concentrate the odor molecules near the sensors. And in the other hand, the aspiration system also helps in the desaturation of the sensors and makes them ready for the next measure.

Once we have a biologically inspired system that perceives the environment and obtain a homogenized and desaturated sample, the next goal is to use different sensors or array of sensors to discriminate more than one odor finding patterns in the environment of different mixtures.

In particular, we aim to apply this system on unmanned vehicles that can locate the source of an odor autonomously or with minimal human intervention, even at outdoors using time and space average, probably following the gradient of the odor trail.

\section{References}

1. Yasuda, G.: A Distributed Autonomous Control Architecture for Synchronization and Coordination of Multiple Robot Systems. In: 2012 Proceedings of Sice. Annual Conference (Sice), pp. 1864-1869 (2012)

2. Lochmatter, T., Martinoli, A., Wani, M.X.C., Casasent, D., Kurgan, L., Hu, T., Hafeez, K.: Simulation Experiments with Bio-Inspired Algorithms for Odor Source Localization in Laminar Wind Flow. In: Proceedings of the Seventh International Conference on Machine Learning and Applications, pp. 437-443 (2008)

3. Lochmatter, T., Martinoli, A., Khatib, O.: Tracking Odor Plumes in a Laminar Wind Field with Bio-inspired Algorithms. In: Khatib, O., Kumar, V., Pappas, G.J. (eds.) Experimental Robotics. STAR, vol. 54, pp. 473-482. Springer, Heidelberg (2009)

4. Huang, Q., Yu, Z., Zhang, W., Duan, X., Huang, Y., Li, K.: Generation of Humanoid Walking Pattern Based on Human Walking Measurement. In: 2008 8th IEEE-Ras International Conference on Humanoid Robots (Humanoids 2008), pp. 99-104 (2008)

5. Goldstein, E.B.: Sensation and Perception. Wadsworth, Cengage Learning, Canada (2010)

6. Browne, C., Stafford, K., Fordham, R.: The use of scent-detection dogs. Irish Veterinary Journal 59, 97-104 (2006)

7. Li, J., Meng, Q., Wang, Y., Zeng, M.: Odor source localization using a mobile robot in outdoor airflow environments with a particle filter algorithm. Autonomous Robots 30, 281-292 (2011) 
8. Loutfi, A., Coradeschi, S., Karlsson, L., Broxvall, M.: Putting Olfaction into Action: Anchoring Symbols to Sensor Data Using Olfaction and Planning (2005)

9. Loutfi, A., Coradeschi, S., Lilienthal, A., Gonzalez, J.: Gas distribution mapping of multiple odour sources using a mobile robot. Robotica 27, 311-319 (2009)

10. Gardner, J., Bartlett, P.: A brief-history of electronic noses. Sensors and Actuators BChemical 18, 211-220 (1994)

11. Gonzalez-Jimenez, J., Monroy, J., Blanco, J.: The Multi-Chamber Electronic Nose-An Improved Olfaction Sensor for Mobile Robotics. Sensors 11, 6145-6164 (2011)

12. Crank, J.: The mathematics of diffusion. Oxford Univerity Press (1976)

13. Pashami, S., Lilienthal, A., Trincavelli, M.: Detecting Changes of a Distant Gas Source with an Array of MOX Gas Sensors. Sensors 12, 16404-16419 (2012)

14. Kowadlo, G., Russell, R.A.: Robot Odor Localization: A Taxonomy and Survey. The International Journal of Robotics Research 27, 869-894 (2008)

15. Miyatani, I., Ishida, H.: Active Stereo Nose: Using Air Curtain to Enhance the Directivity. 2010 IEEE Sensors, 1522-1525 (2010)

16. Lilienthal, A., Duckett, T.: Building gas concentration gridmaps with a mobile robot. Robotics and Autonomous Systems 48, 3-16 (2004)

17. Thomas, L., Xavier, R., Alcherio, M.: Odor Source Localization with Mobile Robots. Bulletin of the Swiss Society for Automatic Control 46, 11-14 (2007)

18. Zhao, K., Scherer, P., Hajiloo, S., Dalton, P.: Effect of anatomy on human nasal air flow and odorant transport patterns: Implications for olfaction. Chemical Senses 29, 365-379 (2004)

19. Craven, B., Paterson, E., Settles, G.: The fluid dynamics of canine olfaction: unique nasal airflow patterns as an explanation of macrosmia. Journal of the Royal Society Interface 7, 933-943 (2010)

20. Reddy, S.S., Ryan, M.W.: Turbinate Dysfunction: Focus on the role of the inferior turbinates in nasal airway obstruction. UTMB, Dept. of Otolaryngology (2003)

21. Hornung, D.E., Mozell, M.M. (eds.): Accessibility of odorant molecules to the receptors, New York (1981)

22. Pearce, T.C., Schiffman, S.S., Nagle, H.T., Gardner, J.W.: Handbook of Machine Olfaction Electronic Nose Technology (2003)

23. Hahn, I., Scherer, P.W., Mozell, M.M.: Velocity profiles measured for airflow through a large-scale model of the human nasal cavity. Modeling Physiol. 75, 2273-2287 (1993)

24. Ganong, W.F.: Review of medical physiology. Prentice Hall (1997)

25. Villarreal, B.L., Hassard, C., Gordillo, J.L.: Finding the Direction of an Odor Source by Using Biologically Inspired Smell System. In: Pavón, J., Duque-Méndez, N.D., FuentesFernández, R. (eds.) IBERAMIA 2012. LNCS, vol. 7637, pp. 551-560. Springer, Heidelberg (2012)

26. Villarreal, B.L., Gordillo, J.L.: Method and artificial olfactive system. IMPI Instituto Mexicano de la Propiedad Intelectual, Mx/a/2012/014508, México (2012)

27. Lilienthal, A., Duckett, T.: A stereo electronic nose for a mobile inspection robot. In: Proceedings of the IEEE International Workshop on Robotic Sensing

28. Villarreal, B.L., Gordillo, J.L.: Directional Aptitude Analysis in Odor Source Localization Techniques for Rescue Robots Applications. In: 10th Mexican International Conference on Artificial Intelligence (MICAI), pp. 109-114 (2011) 